\title{
Quantitative differential interference contrast microscopy based on structured-aperture interference
}

\author{
Xiquan Cui, ${ }^{\text {a) }}$ Matthew Lew, and Changhuei Yang \\ Department of Electrical Engineering, California Institute of Technology, Pasadena, California 91125, USA
}

(Received 21 May 2008; accepted 11 August 2008; published online 4 September 2008)

\begin{abstract}
We report a quantitative differential interference contrast (DIC) microscope based on a structured-aperture (SA) wavefront sensor. Unlike a conventional DIC microscope, the SA-DIC microscope can separate the amplitude and the phase gradient information of the image wavefront, and form quantitative intensity and DIC images of the sample with good resolution; our prototype achieved resolution $\sim 2 \mu \mathrm{m}$. Furthermore, due to the nonpolarization nature of the microscope, we were able to image birefringent samples without artifacts. (C) 2008 American Institute of Physics.
\end{abstract} [DOI: 10.1063/1.2977870]

Differential interference contrast (DIC) microscopes render excellent contrast for optically transparent biological samples without the need of introducing any exogenous contrast agents. Due to this noninvasive nature, DIC microscopes are widely used in biology laboratories. However, conventional DIC microscopes have several limitations. One major disadvantage is that the conventional DIC microscope translates phase variations into amplitude (intensity) variations. Therefore phase variations cannot be easily disentangled from amplitude variations that arise from sample absorption and/or scattering. ${ }^{1}$ In addition, conventional DIC images of birefringent samples can have significant artifacts as the conventional DIC microscope uses polarization in its phase-imaging strategy. ${ }^{2}$

Here we report a quantitative DIC microscopy method based on our recently developed structured-aperture (SA) wavefront sensor. ${ }^{3}$ Benefiting from the unique features of the SA wavefront sensor, our DIC microscope system can separate the amplitude and the phase gradient information of the image wavefront, and form quantitative intensity and DIC images of the sample with good resolution $(\sim 2 \mu \mathrm{m})$. Furthermore, since our system uses unpolarized light and contains no polarization-dependent components, we are able to image birefringent samples, e.g., potato starch storage granules, without artifacts. Finally, unlike most of the recently developed quantitative phase microscopy techniques, ${ }^{4,5}$ this method can be used with a standard microscope light source.

The phase-imaging principle of our quantitative SA based DIC (SA-DIC) microscope can best be understood by taking a closer look at a conventional DIC microscope. In essence, a conventional DIC microscope operates by first creating two identical illumination light fields exploiting polarization selection. The light fields are then laterally displaced (displacement $=a$ ) with respect to each other [along the $x$-direction in Fig. 1(a) example] and are transmitted through the sample. A net phase lag (typically $\pi / 2$ ) is then introduced on one of the transmitted image light fields. Finally, the two light fields are allowed to interfere with each other at the image plane. More simply, the process is equivalent to simply duplicating the transmitted image light field, laterally displacing the copy slightly and interfering the two light fields.

a)Tel.: 626-395-4844. Electronic mail: xiquan@caltech.edu.
Mathematically, this implies that the observed conventional DIC intensity image from a microscope with a magnification factor of $M$ is given by

$$
\begin{aligned}
I_{\mathrm{DIC}}(x, y)= & \left|\psi_{\mathrm{DIC}}(x, y)\right|^{2}=B(x, y)+C(x, y) \\
& \times \sin \{\arg [\psi(x-\Delta / 2, y)]-\arg [\psi(x+\Delta / 2, y)]\} \\
\approx & B(x, y)+C(x, y) \\
& \times\{\arg [\psi(x-\Delta / 2, y)]-\arg [\psi(x+\Delta / 2, y)]\},
\end{aligned}
$$

where $B(x, y)=|[\psi(x-\Delta / 2, y)]|^{2}+|[\psi(x+\Delta / 2, y)]|^{2}, \quad C(x, y)$ $=2|[\psi(x-\Delta / 2, y)]||[\psi(x+\Delta / 2, y)]|$, and $\psi(x, y)$ is the image wavefront as relayed by the microscope for each light field, $\psi_{\text {DIC }}(x, y)$ is the DIC image wavefront, and $\Delta=M a$ is the relative displacement of the images associated with the light fields. The last expression in Eq. (1) is valid only in situations where the phase difference is small.

The phase-related contrast can also be interpreted as a measure of the image's wavefront gradient-defined as $G_{x}(x, y)=k_{x}(x, y) / k_{o}$, where $k_{x}(x, y)$ is the light field's wavenumber along the direction of light fields' displacement ( $x$-direction) and $k_{o}$ is the light field's total wave-
Conventional DIC

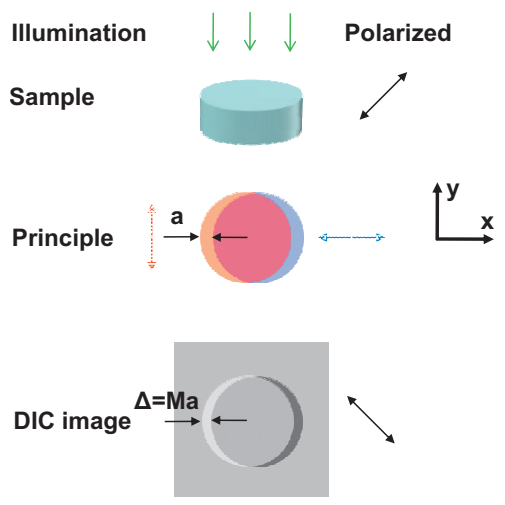

(a)

\section{SA-DIC}
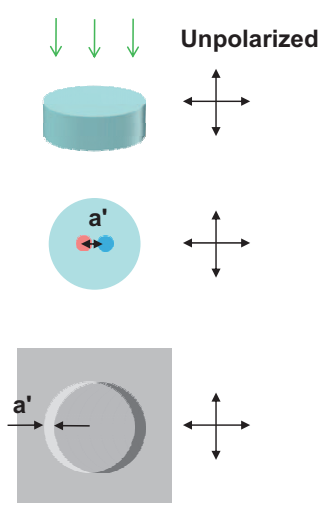

(b)
FIG. 1. (Color) Comparison of conventional DIC and SA-DIC microscopes. (a) conventional DIC microscope operates by interfering slightly displaced duplicate image light fields. (b) SA-DIC microscope operates by interfering light from two adjacent points on the image light field. 
number. Explicitly, $G_{x}(x, y)=\{\arg [\psi(x-\Delta / 2, y)]-\arg [\psi(x$ $+\Delta / 2, y)]\} /\left(k_{o} \Delta\right)$ for this scenario and Eq. (1) can be reexpressed as

$$
I_{\mathrm{DIC}}(x, y) \approx B(x, y)+C(x, y) G_{x}(x, y) k_{o} \Delta .
$$

A similar phase comparison can also be performed by acquiring a simple microscope image of the object and selectively combining and interfering the light fields at two adjacent points of the image (separation $\left.=a^{\prime}\right)$ [Fig. 1(b)]. This is the approach employed in our DIC imaging method.

The SA wavefront sensor is the key component of our DIC microscope system. Our SA comprises of four holes (1 $\mu \mathrm{m}$ diameter, $1 \mu \mathrm{m}$ center-to-center spacing, and the two long axes are in the orthogonal $x$-direction and $y$-direction, respectively) defined in a silver film $(100 \mathrm{~nm}$ thick) above a complementary metal-oxide semiconductor (CMOS) image sensor (Micron MT9V403) [Fig. 2(c)]. The holes and the CMOS sensor are separated by an $80 \mu \mathrm{m}$ thick (d) (measured by a Thorlabs Optical Coherence Tomography system OCMP1300SS) layer of SU-8 resin. By placing the SA wavefront sensor in the image plane of a standard microscope system, the four holes will selectively transmit and combine the light fields from four adjacent points on the image to create an interference pattern on the CMOS sensor. The total transmission of the interference is proportional to the average image intensity at the aperture. In addition to the spacer thickness (d), the offsets $\operatorname{offset}_{x}(x, y)$ and $\operatorname{offset}_{y}(x, y)$ of the zero-order interference spot are related to the net wavefront gradient $G_{x}(x, y)$ and $G_{y}(x, y)$ at the aperture, respectively,

$$
\begin{aligned}
& G_{x}(x, y)=\frac{1}{\sqrt{1+\left[\frac{d}{\operatorname{offset}_{x}(x, y)}\right]^{2}}}, \\
& G_{y}(x, y)=\frac{1}{\sqrt{1+\left[\frac{d}{\operatorname{offset}_{y}(x, y)}\right]^{2}}} .
\end{aligned}
$$

The relative simplicity and absence of image intensityrelated terms makes this a particularly appealing way to measure the wavefront gradient. In addition, this approach allows us to measure the wavefront gradient in both image-plane spatial dimensions simultaneously.

We experimentally determined the exact proportionality of this device by measuring the interference pattern [Fig. 2(d)] as we illuminate the SA with a collimated He-Ne laser beam $(632.8 \mathrm{~nm}$ wavelength, $25 \mathrm{~mm}$ beam diameter, and 4 $\mathrm{mW}$ power from a range of incident angles). We used a leastsquares two-dimensional Gaussian fit to compute the total transmission and the offsets (offset $x$ and offset $_{y}$ ) of the zeroorder spot in both $x$-direction and $y$-direction. Figure 2(b) shows the relationship between offset ( offset ${ }_{y}$ ) of the zeroorder spot and the wavefront gradient $G_{x}\left(G_{y}\right)$. Both curves are approximately linear in our measurement ranges. This is consistent with the geometric optics prediction: offset $_{x}$ $=d \tan (\theta) \approx d G_{x}$, and similarly for offset $y$, where $\theta$ is the incident angle of the laser beam and when the angle is small. Our experimentally measured proportionality from Fig. 2(b) was $70 \mu \mathrm{m}$ while the predicted value from thickness $d$ measurement was $80 \mu \mathrm{m}$. Finally, we note that this SA wave- (a)

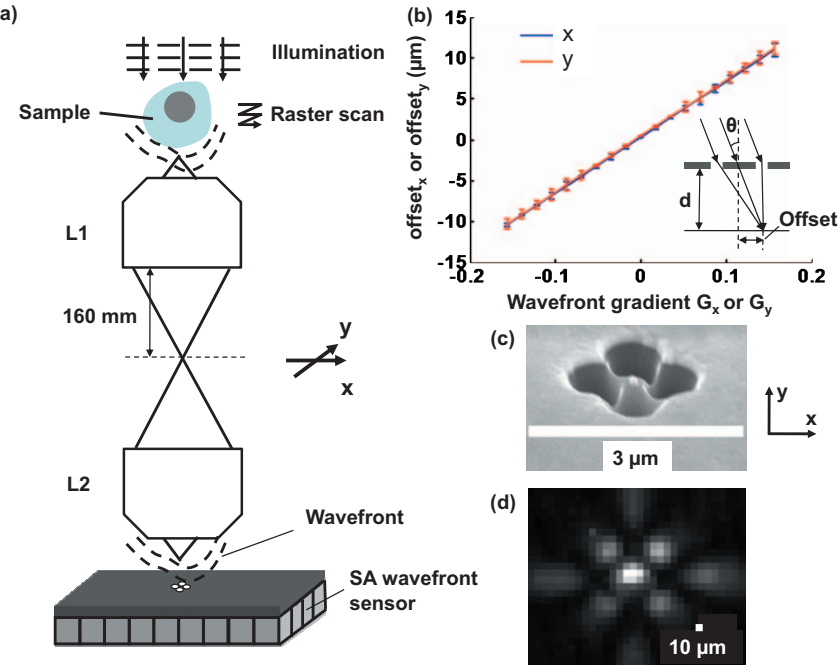

FIG. 2. (Color) SA-DIC microscope and SA wavefront sensor. (a) Configuration of the SA-DIC microscope; (b) the offset in blue (offset $_{y}$ in red) of the zero-order interference spot of the SA is linearly proportional to the wavefront gradient $G_{x}\left(G_{y}\right)$ along the $x$ - $(y$-) direction in our measurement range; (c) scanning electron microscope image of the SA defined on the silver film; and (d) the interference pattern of the SA recorded by the CMOS image sensor

front sensor works with broadband light sources as well, as the zero-order interference spots coincide spatially for all wavelengths.

The experimental scheme of our SA-DIC microscope is shown in Fig. 2(a). We aligned two $20 \times$ objective lenses (Newport M-20X) such that their rear conjugate planes (160 $\mathrm{mm}$ behind the objective lens) overlap. We placed the sample at the front conjugate plane of the top objective (L1), and illuminated it with a collimated white light (halogen lamp, $200 \mathrm{~mW} / \mathrm{cm}^{2}$ ). Since the microscope system was symmetric, it formed a 1:1 image of the sample at the front conjugate plane of the bottom objective (L2); this image was equal to the convolution of the input sample light field with the PSF of the microscope system. Our SA wavefront sensor was placed at the center of the image plane. We raster scanned (by two Newport CMH-25CCCL actuators) the sample in the $x-y$ plane to complete the mapping of the intensity and wavefront gradient of the image. During the imaging process, the illumination, the optical system, and the sensor were fixed with respect to each other. This allowed stable and precise measurement with our simple optical system.

To demonstrate the quantitative DIC sensing capability of our system and its application in biological imaging, we chose a starfish embryo (Carolina Scientific) as a test sample. Figure 3(a) shows a microscope (Olympus BX41) image of the sample acquired with a $10 \times$ objective. Figure $3(b)$ is the corresponding image under a standard DIC microscope (Zeiss Axioplan, $40 \times$ objective). Figures 3(c)-3(e) show the intensity, $X$ and $Y$ DIC phase images acquired by our microscope with a single scan. The spatial sampling step size was $0.5 \mu \mathrm{m}$ in both $x$-direction and $y$-direction. The exposure time associated with each sampling point was $8 \mathrm{~ms}$.

The conventional transmission microscope image and the SA-DIC intensity image are consistent with each other. On the other hand, our SA-DIC phase images do appear to be different from the conventional DIC image. This is because, while our SA-DIC phase images purely map the wavefront gradients, the conventional DIC image contains 

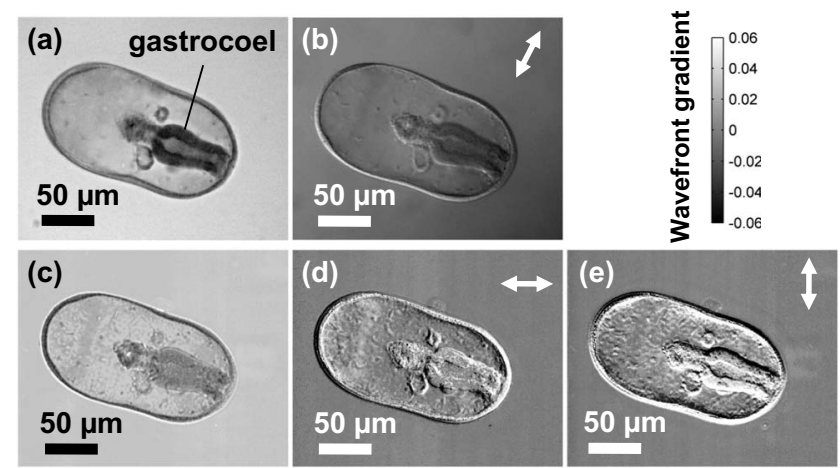

FIG. 3. Images of starfish embryo. (a) Conventional transmission microscope image; (b) conventional DIC microscope image; the (c) intensity, (d) $X$ DIC; and (e) $Y$ DIC phase images from the SA-DIC microscope. (Double arrows represent the DIC directions).

intensity image variations as well. This distinction is particularly apparent when we compare the embryo's gastrocoel for all of the images. The image intensity associated with that region is low and the region appears darker in the conventional DIC image. In comparison, the corresponding areas of our SA-DIC phase images do not appear darker because they are pure phase maps. Finally, we note that the SA-DIC phase and amplitude images are also an improvement over conventional DIC images in that they are quantitative maps. The wavefront gradient sensitivity of our prototype operating in the above described experimental conditions is approximately $4 \mathrm{mrad}$; the sensitivity can be improved by using a better sensor platform, increasing measurement time and/or increasing the illumination intensity. ${ }^{3}$

Our system's ability to image birefringence samples properly is yet another advantage. Birefringent objects, such as the potato starch storage granules, can alter the polarization of the two displaced light fields in a conventional DIC microscope, such that the subsequent combination of the two fields is no longer describable by Eq. (1). This can give rise to Maltese-cross-like pattern artifacts in the resulting conventional DIC images [Fig. 4(b)]. ${ }^{2}$ Since our SA-DIC micro-

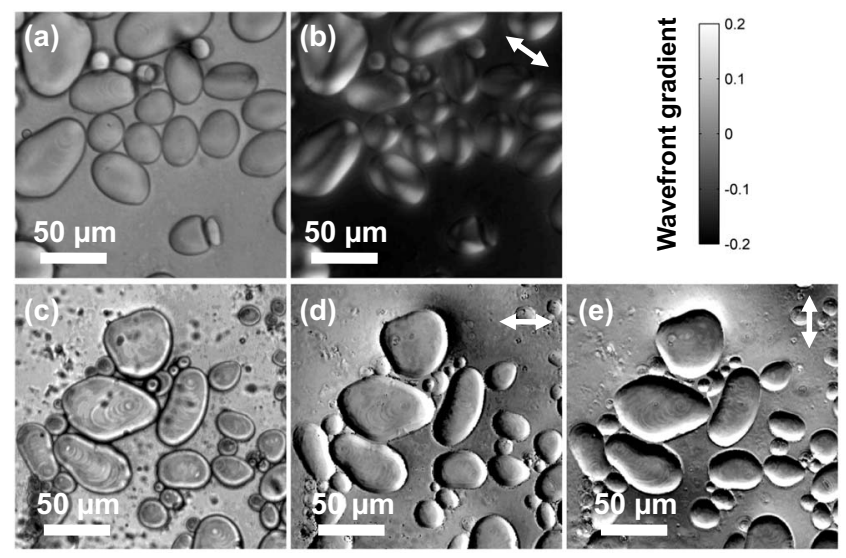

FIG. 4. Images of potato starch storage granules in microscope immersion oil. (a) Conventional transmission microscope image; (b) Maltese-cross-like pattern artifacts in the conventional DIC image; the (c) intensity, and artifact-free (d) $X$ DIC; and (e) $Y$ DIC phase images from the SA-DIC microscope. (Double arrows represent the DIC directions). scope uses unpolarized illumination and does not rely on polarization for image processing, it can image birefringent samples without artifacts, as shown in Figs. 4(d) and 4(e). It is also worth noting that the dark absorption spots of the starch granules in the center of the intensity images [Fig. 4(c)] do not appear in our SA-DIC phase images [Figs. 4(d) and 4(e)]. This is another clear indication that our microscope can separate the intensity variations of the image wavefront from the phase variations.

The aperture size impacts on several aspects of the system performance and is, therefore, an important design consideration worth discussing. The SA-DIC microscope resolution is limited by the numerical aperture of the collection optics or the aperture size divided by the system's magnification $(M)$. Our prototype is limited by the latter and has a resolution equal to the aperture size $(2 \mu \mathrm{m})$ (our imaging system has $M=1)$. We can create a system that is limited by the numerical aperture of the collection optics by either increasing $M$ or decreasing the aperture size. A smaller aperture can lead to a decreased sensitivity of the wavefront gradient measurement for two reasons. First, a smaller aperture will lead to a broader interference pattern, which negatively impacts on our ability to determine the offsets of the zeroorder intereference spot. ${ }^{6}$ Second, a smaller aperture will transmit less light and therefore lead to an overall decrease in the detected signal.

In conclusion, we have demonstrated a high-resolution and artifact-free quantitative DIC microscopy method based on the SA wavefront sensor. The method can simultaneously generate one intensity and two orthogonal wavefront gradient images. Unlike a conventional DIC microscope, SA-DIC is capable of imaging birefringent samples without artifactual image errors. The relatively long image acquisition time of our prototype is the only significant shortcoming. However, we fully expect that the imaging speed can be dramatically increased by using more sensitive detectors, e.g., avalanche photodiode array, employing faster scanning system, and/or parallelizing the imaging process. ${ }^{7,8}$

The authors acknowledge financial support from the Defense Advanced Research Projects Agency Center for Optofluidic Integration. We thank Professor Paul Sternberg, Dr. Ying Zhou, Jigang Wu, Guoan Zheng, and Emily McDowell for their technical assistance and enlightening discussions.

\footnotetext{
${ }^{1}$ C. J. Cogswell and C. J. R. Sheppard, J. Microsc. 165, 81 (1992).

${ }^{2}$ See http://micro.magnet.fsu.edu/primer/techniques/dic/ dicphasecomparison.html for the birefringent artifacts in conventional DIC microscope images.

${ }^{3}$ M. Lew, X. Cui, X. Heng, and C. Yang, Opt. Lett. 32, 2963 (2007).

${ }^{4}$ P. Marquet, B. Rappaz, P. J. Magistretti, E. Cuche, Y. Emery, T. Colomb, and C. Depeursinge, Opt. Lett. 30, 468 (2005).

${ }^{5}$ W. Choi, C. Fang-Yen, K. Badizadegan, S. Oh, N. Lue, R. R. Dasari, and M. S. Feld, Nat. Methods 4, 717 (2007).

${ }^{6}$ R. E. Thompson, D. R. Larson, and W. W. Webb, Biophys. J. 82, 2775 (2002).

${ }^{7}$ X. Heng, D. Erickson, L. R. Baugh, Z. Yaqoob, P. W. Sternberg, D. Psaltis, and C. H. Yang, Lab Chip 6, 1274 (2006).

${ }^{8}$ X. Cui, X. Heng, J. Wu, Z. Yaqoob, A. Scherer, D. Psaltis, and C. Yang, Opt. Lett. 31, 3161 (2006).
} 